\title{
Using Whole-Genome Sequencing to Identify Recent Introductions and Track the Spread of Candida auris in the United States
}

Chow NA ${ }^{1}$, Gade $L^{1}$, Tsay $S^{1}$, Welsh $R^{1}$, Forsberg $K^{1}$, Adams, Eleanor ${ }^{2}$, Southwick $K^{2}$, Greenko $\mathrm{J}^{2}$, Fernandez $\mathrm{R}^{2}$, Chiller $\mathrm{T}^{1}$, Jackson $\mathrm{B}^{1}$, Vallabhaneni $\mathrm{S}^{1}$, and Litvintseva AP ${ }^{1}$ Mycotic Diseases Branch, Centers for Disease Control and Prevention, Atlanta GA USA ${ }^{2}$ New York State Department of Health, NY USA

OBJECTIVES: Candida auris has emerged as a multidrug-resistant yeast in healthcare settings throughout the world and recently in the United States. The fungus can cause invasive infections associated with high mortality, colonize patients and contacts, and persist on environmental surfaces for months. We characterized the molecular epidemiology of $C$. auris in the United States to inform understanding of transmission patterns and outbreak response.

METHODS: We performed WGS on 300 C. auris isolates obtained from clinical and screening cases identified within ten U.S. states: California (CA), Connecticut (CT), Florida (FL), Illinois (IL), Indiana (IN), Maryland (MD), Massachusetts (MA), New Jersey (NJ), New York (NY), and Oklahoma (OK). Pairwise SNP comparisons of multiple isolates from the same patient and between patients within a facility with active transmission were examined.

RESULTS: We found all four known C. auris populations represented in the United States. Patient isolates from CA, CT, MA, MD, NJ, NY, and OK clustered with isolates from India and Pakistan (South Asian clade). Patient isolates from FL, IL, and MA clustered with those from Venezuela and Colombia (South American clade). The patient isolate from IN clustered with those from South Africa, and two people from NY had isolates that clustered with those from Japan and South Korea (East Asian clade). Five patients had been hospitalized in a country with known $C$. auris transmission before $C$. auris identification. Little intra-host genetic diversity of $C$. auris was observed within each patient. Patient isolates within each facility with active transmission had similarly limited diversity.
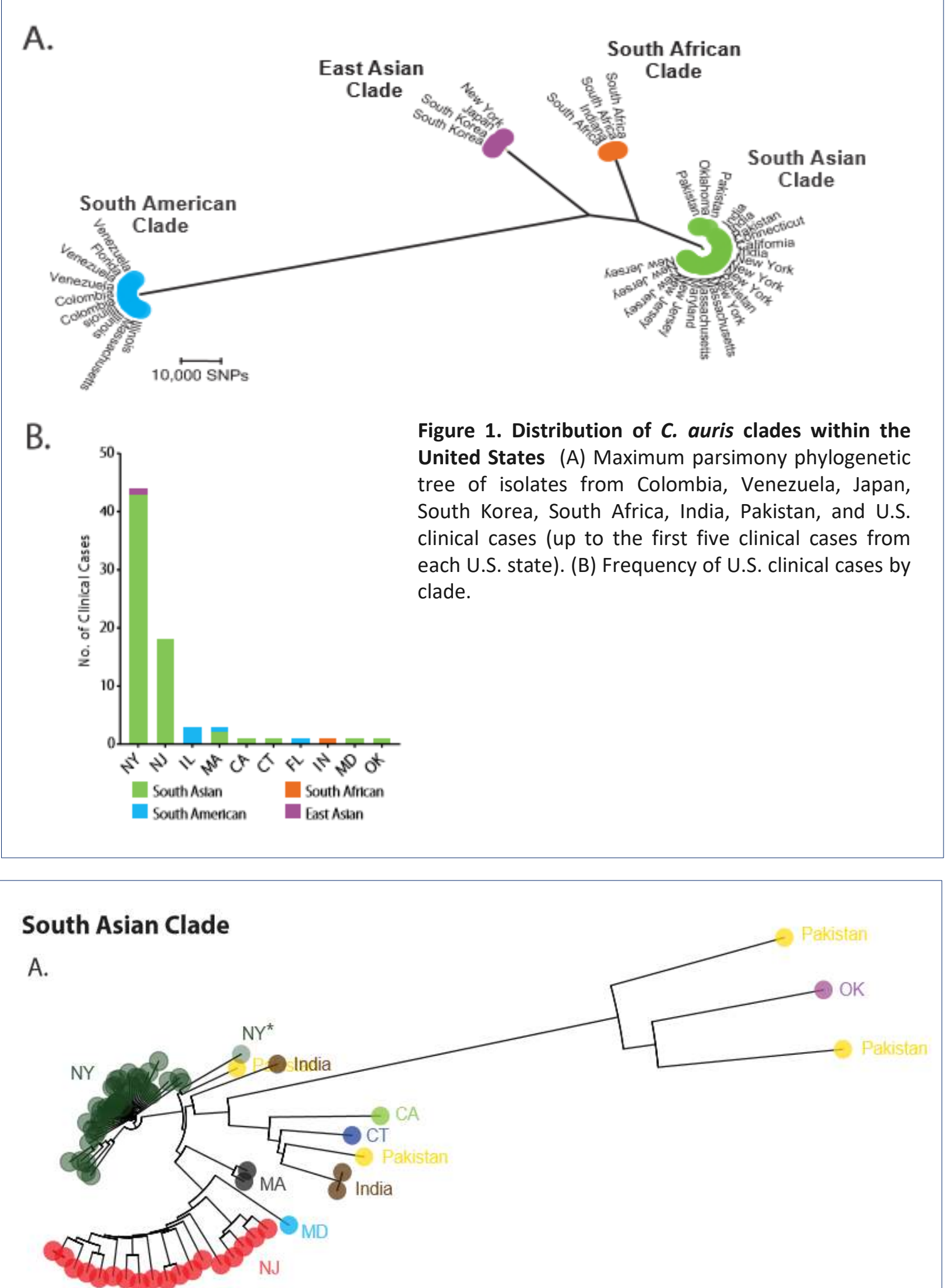

Figure 2. Identification of genetically distinct $C$. auris strains in different U.S. states. (A) Neighbor-joining phylogenetic tree of the South Asian clade examining isolates from clinical cases from seven U.S. states, India, and Pakistan. Circles at nodes indicate separations with a bootstrap value $\geq 90 \%$.
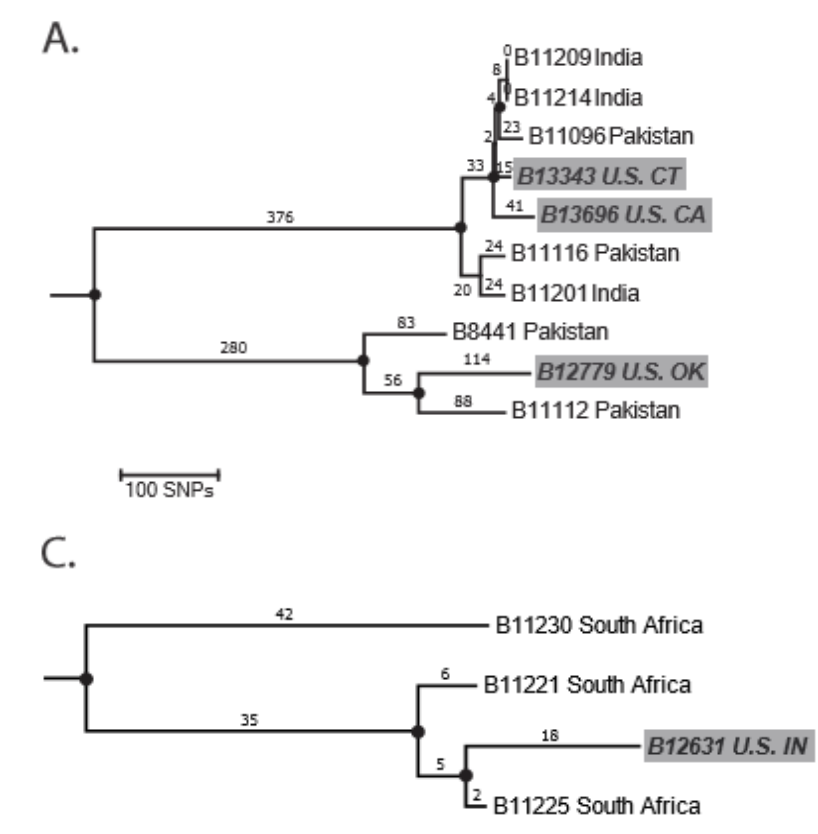

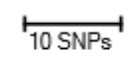

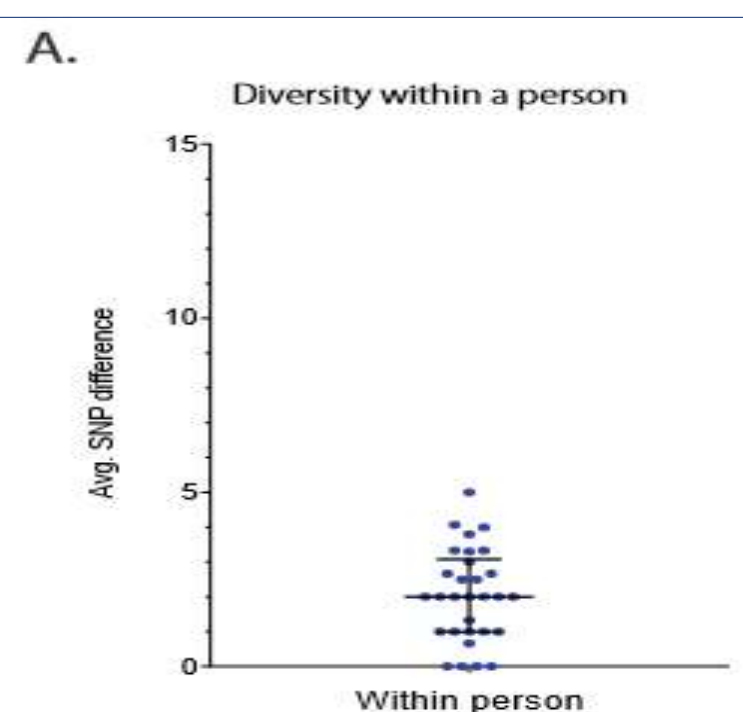

Figure 4. Description of $C$. auris genetic diversity among U.S cases during transmission. (A) Average SNP difference among multiple isolates from the same person, for 23 U.S. cases. (B) SNP differences for each pairwise comparison among 10 cases at a NJ facility experiencing an outbreak. (C) SNP differences for each pairwise comparison among all NY cases, excluding the 2013 case, and among the epidemiologically-linked pairwise comparisons, NY. Light blue diamond indicates pairwise comparisons with a specific NY screening case.

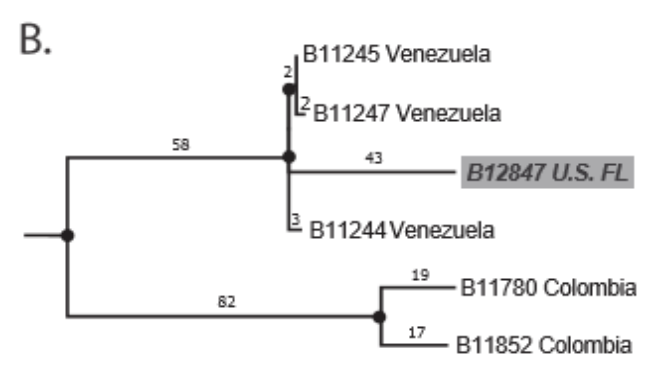

20 SNPS

Figure 3. Identification of five U.S. travelrelated $C$. auris cases. Phylogenetic trees of the South Asian (A), South American (B), and the South Asian (A), South American (B), and
African (C) clades examining isolates from clinical cases known to have received medical care abroad prior to diagnosis of $C$. auris infection in the U.S. Circles at nodes indicate separations with a bootstrap value $\geq 90 \%$.

B.

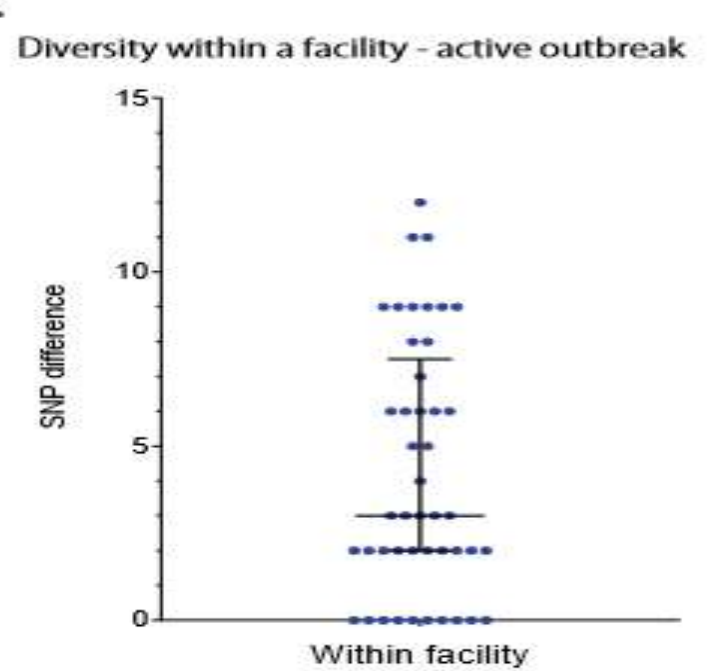

C.

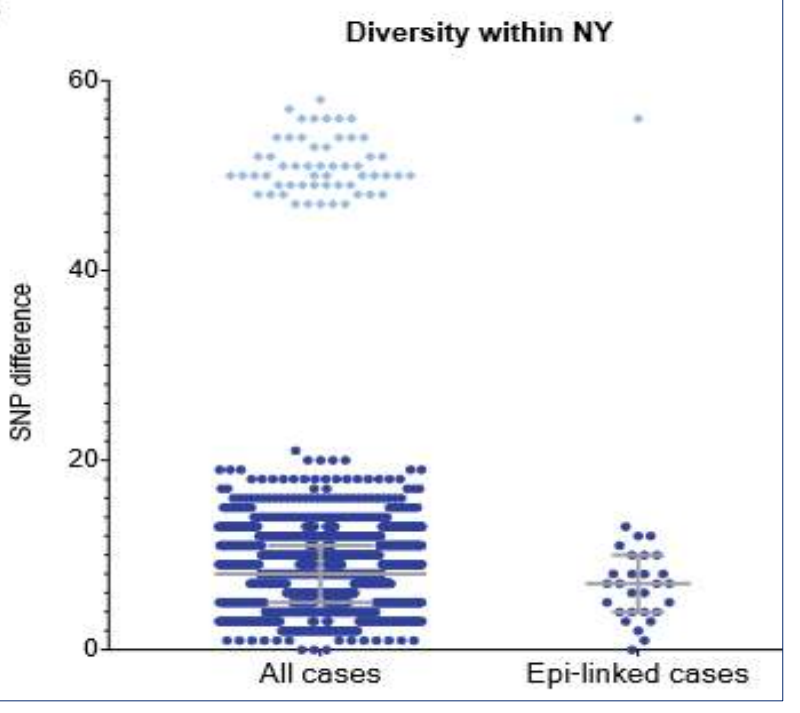

CONCLUSIONS: WGS has helped public health officials define, track, and monitor $C$. auris outbreaks in the United States. These findings suggest multiple recent introductions and support heightened monitoring for $C$. auris in patients recently hospitalized in a country with known transmission. Minimal diversity observed within and between patients in a facility further suggests recent transmission and need for infection control measures.

CONTACT:

Nancy Chow (ynl3@cdc.gov) Mycotic Diseases Branch. CDC 